\title{
UNEXPECTED FAILURE OF FERTILIZATION IN CONVENTIONAL IVF CYCLES: RE-INSEMINATION BY ICSI OR INDICATION FOR ICSI IN SUBSEQUENT CYCLES?
}

\author{
Ehab M. Soliman, MD and Amani T. Makkar, MSc \\ Al-Salam Center for Assisted Reproduction, Mohandeseen, Cairo, Egypt
}

\section{ABSTRACT}

\begin{abstract}
Objective : To compare the results of cycles with complete failure of fertilization in which rescue ICSI was performed with a matched control group of ICSI cycles, both in terms of fertilization and pregnancy outcome and to answer the question whether rescue ICSI is worth trying.
\end{abstract}

Design : Retrospective clinical study.

Setting : Private assisted reproduction unit.

Material and Methods : The study included 24 patients (group I) undergoing a first trial conventional IVF for non-male factor infertility, and in whom unexpected total failure of fertilization was discovered. A rescue ICSI procedure was performed where at least three metaphase II (MII) oocytes were available. A control group (group II) was retrospectively selccted and included 27 couples undergoing ICSI for male factor infertility during the same period. Patients were matched for agc, duration of infertility, type of ovarian stimulation and number of oocytes retrieved.

Results: The fertilization rate, expressed as the percentage of 2PN oocytes per injected oocytes (47\% vs. $70.3 \%$ ), was significantly higher in group II ( $\mathrm{P}<0.05)$. The proportion of grade I embryos, however, assessed on day 2 was comparable in both groups ( $47.2 \%$ vs. $51.2 \%)$. The percentage of cycles with transfer (66.6\% vs. $92.6 \%)$ and the mean number of embryos transferred ( 1.66 vs. 2.9) were significantly higher in the control group $(P<0.05)$. Nine pregnancies were obtained in the control group (clinical pregnancy rate of $33.3 \%$ per cycle and $36 \%$ per (ransfer). In group I patients with rescue ICSI, no pregnancies were obtained.

Conclusion: Rescue ICSI performed on 1-day-old unfertilized oocytes, although resulting in fertilization, gives poor results in terms of pregnancy. Ageing of the oocytcs presumably affects the developmental capacity of the embryo. We recommend that resuce ICSI of 1-day-old oocytes be limited as a diagnostic method that serves as an indication for ICSI in subsequent cycles.

Key words : Fertilization failure, reinsemination, IVF, rescue ICSI.

\section{INTRODUCTION}

The rates of fertilization with conventional IVF has been reported between $60 \%$ and $70 \%$ in spite of this, the results can vary until reaching the point of complete failure (1). Complete failure of fertilization after IVF, in cases with normal semen parameters, is a frustrating experience for the infertile couple as well as for the infertility team. Sperm defects ${ }^{(2)}$, disturbances in sperm oocyte interaction and oocyte abnormalities have all been proposed as possible causes of failed fertilization after IVF ${ }^{(3,4)}$. From the beginning of IVF, various procedures to salvage the lost cycles have been attempted. Reinsemination of the unfertilized oocytes 24 hours after oocyte retrieval has been tried (5). After the advent of micromanipulation techniques, ICSI was introduced to rescue oocytes after unsuccessful IVF (6,7,8). Although pregnancies have been reported after rescue of

Corresponding author : Ehab Mohamed Soliman, 13, El-Batal Ahmed Abdel Aziz st, Mohandeseen, 12411, Cairo, Egypt. Fax: (202) 3030140 - E-mail: emmahs@yahoo.com. 
failed fertilization, most reports were relatively inconclusive.

The aim of this study was to compare the results of cycles with complete failure of fertilization in which rescue ICSI was performed with a matched control group of ICSI cycles, both in terms of fertilization and pregnancy outcome and to answer the question whether recue ICSI is worth trying.

\section{MATERIALS \& METHODS}

This is a retrospective clinical trial that included 24 patients (group I) undergoing a first trial conventional IVF for non-male factor infertility, and in whom unexpected total failure of fertilization was discovered during the $\mathrm{PN}$ check 16-18 hours post-insemination. A rescue ICSI procedure was performed in these cycles where at least three metaphase II (MII) oocytes were available and after an informed written consent from the patients. All couples in the IVF group met standard criteria for admission to IVF, including normal semen parameters as assessed by published criteria (9). Indications for IVF were mainly unexplained infertility, endometriosis, tubal obstruction and/or adhesions.

A control group (group II) was retrospectively selected and included 27 couples undergoing ICSI for male factor infertility during the same period. Patients were matched for age, duration of infertility, type of ovarian stimulation and number of oocytes retrieved. Indications for ICSI were moderate or severe oligo/astheno/teratospermia, either singly or in combination. Patients undergoing ICSI for necrospermia, functional and/or obstructive azoospermia were excluded.

Ovarian stimulation was carried out using the mid-luteal down-regulation protocol. Diphereline
(Decapeptyl®B), Beaufour Ipsen, France) and purified urinary FSH or HMG (Fostimon ${ }^{\circledR}$ or Merional ${ }^{\circledR}$, IBSA, Swiss) were used. Oocyte retrieval was done 34-36 hours following 10.000 I.U. HCG (Choragon®, Ferring, Germany), and the luteal phase was supplemented with vaginal micronized progesterone.

Sperms were prepared for insemination using the discontinuous density gradient (PureSperm ${ }^{\circledR}$, Nidacon, Sweden). For conventional IVF, oocytes were inseminated with a calculated volume of sperm suspension which resulted in a concentration of -100.000 progressively motile sperms as described (10). For the ICSI procedure, a previously described protocol was followed (11). For oocytes in group I, fresh sperms or motile sperms retrieved from the IVF incubation plate were used for rescue ICSI.

After incubation for 16-18 hours, the oocytes were checked for the presence of pronuclei as proof of fertilization. Embryo culture was followed in sequential media under standard conditions. fertilization was considered to be normal when two individual or fragmented polar bodies and two pronuclei were present. The developmental stages and morphological aspects of embryos were evaluated 48 hours after ICSI according to published criteria (12).

\section{RESULTS}

There was no statistical difference in age and duration of infertility between both groups. The mean age was 31.6 nd 31.3 years and the mean duration of infertility was 5.4 and 5.1 years, respectively. No significant differences in oocyte recovery were observed between the two grups (245 vs. 266 oocytes), or in the percentage of injected MII oocytes $(92.2 \%$ vs. $88 \%$ ) (Table I). 
The fertilization rate, expressed as the percentage of 2PN oocytes per successfully injected oocytes (47\% vs. $70.3 \%)$ and as the percentage of $2-4$ cell embryos on day 2 after ICSI ( $51.8 \%$ vs. $72.5 \%$ ), was significantly higher in group II $(\mathrm{P}<0.05)$. The proportion of grade I embryos, however, assessed on day 2 , was comparable in both groups $(47.2 \%$ vs. $51.2 \%$ ) (Table I).

The percentage of cycles with transfer $(66.6 \%$ vs. $92.6 \%$ ) and the mean number of embryos transferred (1.66 vs. 2.9$)$ were significantly higher in the control group $(\mathrm{P}<0.05)$ (Table II). Nine pregnancies were obtained in the control group (clinical pregnancy rate of $33.3 \%$ per cycle and $36 \%$ per transfer). In group I patients with rescue ICSI, no pregnancies were obtained.

Of the 24 patients in the rescue ICSI group, 14 were subsequently treated by ICSI and 6 became pregnant, including 2 sets of twins and one set of triplets.

Table I : Outcome of ICSI of day 1-old-oocytes (group I) and fresh oocytes (group II)

\begin{tabular}{|l|c|c|}
\hline & Group I & Group II \\
\hline No. patients & 24 & 27 \\
No. oocytes & 245 & 266 \\
No. metaphase Hoocytes $(\%)$ & $226(92.2)$ & $233(88)$ \\
No. 2PN oocytes $(\%)$ & $106(47)$ & $164(70.3)^{*}$ \\
No. 2-4 cells embryos $(\%)$ & $55(51.8)$ & $119(72.5)^{*}$ \\
No. grade I embryos $(\%)$ & $26(47.2)$ & $61(51.2)$ \\
\hline
\end{tabular}

$* \mathrm{P}<0.05$

$\mathrm{PN}$ - pronuclei

Table II : Pregnancy outcome after ICSI of 1-day-old oocytes (group I) and fresh oocytes (group II)

\begin{tabular}{|l|c|c|}
\hline & Group I & Group II \\
\hline Mean no, of embryos transferred & 1.66 & $2.90^{*}$ \\
No. of transfers/cycles $(\%)$ & $16 / 24(66.6)$ & $25 / 27(92.6)^{*}$ \\
Clinical pregnancy/cycle $(\%)^{\mathrm{a}}$ & 0 & $9 / 27(33.3)$ \\
Clinical pregnancy/transfer $(\%)^{\mathrm{a}}$ & 0 & $9 / 25(36)$ \\
\hline
\end{tabular}

$* \mathrm{P}<0.05$

a confirmed by ultrasound 


\section{DISCUSSION}

ICSI was first applied in cases of severe male factor infertility in which conventional IVF would be expected to fail or to give poor outcomes (11). More recently, however, ICSI has been increasingly used in cases in which conventional IVF would not necessarily be efficient. In such cases, ICSI is often preferred because of its reliability in terms of achieving high fertilization rates despite the occurrence and severity of different sperm abnormalities, some of which are not detected by standard semen examinations. In fact, conventional IVF can give unwelcome surprises and complete failure of fertilization, in some cases with normal semen parameters. This is a frustrating experience for the infertile couple as well as for the infertility team.

Because fertilization at IVF is considered the ultimate proof of the fertilizing capacity of the sperm, there is an intuitive tendency conversly assume that failure of fertilization is due to some sperm defect. When the sperm is normal according to the classical criteria, and as yet undisclosed sperm defect is presumed to be present ${ }^{(2)}$. Poor or absent binding of sperm to the zona pellucida is readily ascribed to abnormalities of the sperm (13). There are, however, no firm data to substantiate this hypothesis. Pregnancies either spontaneous or in subsequent IVF cycles do occur in patients with previous unexplained failure of fertilization, which suggests that occult sperm deficiencies, if they exist, are transient. Oocyte-borne failures of conventional IVF are also known, and the corresponding oocyte abnormalities are even more difficult to detect. Bedford et al., ${ }^{(3)}$ demonstrated that inability of apparently competent sperm to penetrate the zona is often associated with ooplasmic anomalies such as refractile bodies, extra groups of chromosomes, chromatin rings or masses as well as one or more pronuclei with one or no polar bodies. Further downstream, a number of defects in the fertilization process such as sperm fusion with oolemma, nuclear decondnsation or formation of male pronucleus and failed oocyte activation have been reported ${ }^{(14,15,16)}$. Besides a disordered sperm-oocyte interaction, there are a number of developmental defects of the oocyte which can impede fertilization and/or the reproductive outcome. Spontaneous cytoplasmic activation resulting in premature cortical granule loss and zona pellucida hardening (17) or cytoplasmic degeneration, manifested by refractile bodies, can affect fertilization (18). Arrest or asynchrony of cytoplasmic maturation can have an impact on the reproductive outcome of fertilized oocytes $(19)$.

Before ICSI became available, it was routine practice to re-inseminate 1-day-old oocytes that had failed to fertilize, usually with poor outcomes (20). With the introduction of ICSI, several investigators $(8,21,22,23)$ reported successful rescue of 1-day-old oocytes by ICSI after fertilization failure. Fertilization and subsequent pregnancy rates from such resuce attempts varied from 24 to $48 \%$ and 0 to $20 \%$ respectively. In our study, following rescue ICSI after 18-20 hours, a fertilization rate of $47 \%$ was achieved, but no pregnancies resulted.

Fertilization of oocytes by rescue ICSI indicates that the intrinsic fertilizing capacity of the sperm is intact in most cases. The poor outcome in terms of pregnancy indicates that some intrinsic or developmental defect of the oocyte is likely to be present in cases of failed fertilization after IVF with normal sperm. This hypothesis is supported 
by the success of oocyte donation in cases of previously and repeatedly failed fertilization (24). Ageing of the oocyte due to delayed fertilization presumably is the most important reason for the poor outcome. The time interval between oocyte retrieval and fertilization is critical for an optimal embryo development. While incubation of oocytes for a period of 3 hours before ICSI improves the fertilization rate and the embryo quality ${ }^{(25)}$, an incubation period of 8 hours seems to be the limit beyond which the outcome of ICSI will be affected (26). Nagy et al, (27) demonstrated that the fertilization decreased and that the incidence of abnormal fertilization increased with oocyte ageing. Recently, Chen and Kattera ${ }^{(28)}$ reported high fertilization and pregnancy rates (70 and $48 \%$ respectively), comparable to ICSI performed as the first choice, in cases in which rescue ICSI was done as early as 6 hours after conventional IVF. Rescue ICSI was performed only in patients in whom all oocytes failed to be fertilized. These data are important because they provide, for the first time, an efficient back-up option for cases in which over-estimation of gamete fertilizing ability leads to erroneous indication of conventional IVF with subsequent fertilization failure. Paradoxically, the success of rescue ICSI can be expected to reduce the indication for ICSI as the first therapeutic choice. This can limit the additional risk, cost and laboratory load ICSI represents in comparison with conventional IVF ${ }^{(29)}$.

In conclusion, rescue ICSI performed on 1-day-old unfertilized oocytes, although resulting in fertilization, gives poor results in terms of pregnancy. Ageing of the oocytes presumably affects the developmental capacity of the embryo. Until further studies confirm the efficacy and safety of rescue ICSI performed as early as 6 hours after in-vitro insemination, we recommend that rescue ICSI of 1-day-old oocytes be limited as a diagnostic method that serves as an indication for ICSI in subsequent cycles.

\section{REFERENCES}

1. Tsirigoitis M, Nicholson N, Taranissi M, Bennet V, Pelekanos M, Craft I. Late intracytoplasmatic sperm injection in unexpected failed fertilization in vitro: diagnostic or therapeutic? Fertil Steril 1995; 4: 816-9.

2. Liu DY and Baker HW. Defective sperm-zona pellucida interaction: a major cause of failure of fertilization in clinical in-vitro fertilization. Hum Reprod 2000; 15: 702-8.

3- Bedford JM and Kim HH. Sperm/egg binding patterns and oocyte cytology in retrospective analysis of fertilization failure in vitro. Hum Reprod $1993 ; 8: 453-63$.

4- Van Blerkom J, Davis PW, Merriam J. A retrospective analysis of unfertilized and presumed parthenogenetically activated human oocytes demonstrates a high frequency of sperm penetration. Hum Reprod 1994; 9: 2381-88.

5- Calderon G, Veiga A, Penella J, Barri PN. Two years of assisted fertilization by partial zona dissection in male factor infertility. Fertil Stcril 1993; 60: 105-9.

6- Nagy ZP, Joris H, Liu J, Staessen C, Deverocy P, Van Steirteghem AC. Intracytoplasmic single sperm injection of 1-day-old unfertilized human oocytes. Hum Reprod 1993; 8: 2180-84.

7- Loong EP, Chiu TT, Haines CJ. Pregnancies from subzonal insemination of unfertilized oocytes after conventional IVF. Chin Med J 1997; 110: 479-81.

8- Yuzpe A, Liu Z, Fluker M. Rescue intracytoplasmatic sperm injection (ICSI)-salvaging in vitro fertilization (IVF) cycles after total or near-total fertilization failure. Fertil Steril 2000; 6: 1115-9.

9- World Health Organization WHO laboratory manual for the examination of human semen and sperm-cervical mucus interaction, $3^{\text {rd }}$ edn Cambridge University Press, Cambridge, p. 44. 
10- Enginsu M, Pieters M, Dumoulin J et al. Male factor as determinant of in-vitro fertilization outcome. Hum reprod 1992; 7: 1136-40.

11- Van Steirteghem A, Nagy Z, Joris H, Liu J, Staessen $\mathrm{C}$, Smitz $\mathrm{J}$ et al. High fertilization and implantation rates after intracytoplasmic sperm injection. Hum reprod 1993; 8: 1061-6.

12- steer $\mathrm{C}$, mills $\mathrm{C}$, Tan $\mathrm{S}$. The cumulative embryo score: a predictive embryo scoring technique to select the optimal number of embryos to transfer in an in-vitro fertilization and embryo transfer programme. Hum Reprod 1992; 7: 117-9.

13- Liu DY, Lopata A, Johnson WI, Baker HW. Human sperm-zona pellucida binding, sperm characteristics and in-vitro fertilization. hum Reprod 1989; 4: 696-701.

14- Dozortsev D, De Sutter P, Dhont M. Behaviour of spermatozoa in human oocytes displaying no or one pronucleus after intracytoplasmic sperm injection. Hum Reprod 1994; 9: 2139-44.

15- Flaherty SP, Payne D, swann NJ, Mattews CD. Aetiology of failed and abnormal fertilization after intracytoplasmic sperm injection. Hum Reprod 1995; 10: 2623-9.

16- Palermo GD, Avrech OM, Colombero LT, Wu H, Wolny YM, Fissore RA et al. Human sperm cytosolic factor triggers $\mathrm{Ca}^{2}+$ oscillations and overcomes activation failure of mammalian oocytes. Mol Hum Reprod 1997; 3: 367-74.

17- Ducibella T. Dubey A, Gross V, Emmi A, Penzias AS, Layman $L$ et al. A zona biochemical change and spontaneous cortical granule loss in eggs that fail to fertilize in vitro fertilization. Fertil Steril 1995; 64: 1154-61.

18- Serhal PF, Ranieri DM, Kinis A, Marchant S, Davies M, Khadum IM. Oocyte morphology predicts outcome of intracyloplasmic sperm injection. Hum Reprod 1997; 12: 1267-70.

19- Kubiak JZ. Mouse oocytes gradually develop the capacity for activation during the metaphase. II arrest. Dev Biol 1989; 136: 537-45.

20- Boldt J, Howe AM, Butler WJ, Mc Donough PG, Padilla SL. The value of oocyte reinsemination in human in vitro fertilization. Fertil Steril 1987; 48: 617-23.

21- Sjogren A, Lundin K, Hamberger L. Intracytoplasmic sperm injection of 1-day-old oocytes after fertilization failure [letter]. Hum Reprod 1995; 10: 974-5.

22- Morton PC, Yoder CS, Tucker MJ, Wright G, Brockmah WD, Kort HI. Reinsemination by intracyloplasmic sperm injection of 1-day-old oocytes after complete conventional fertilization failure. Fertil Steril 1997; 68: 488-91.

23- Kuczynski W, Dhont M, Grygoruk C, Pietrewicz P, Redzko S, Szamatowicz M. Rescue ICSI of unfertilized oocytes after IVF. Hum Reprod 2002; 17: 2423-27.

24- Borini A, Bafaro MG, Bianchi L, violini F, Bonu MA, Flamingi C. Oocytr donation programme: results obtained with intracytoplasmic sperm injection in cases of severe male factor infertility or previous failed fertilization. Hum Reprod 1996; 11 : $548-50$

25- Bienzi L, Ubaldi F, Anniballo R, Cerulo G, Greco E. Preincubation of human oocytes may improve fertilization and embryo quality after intracytoplasmic sperm injection. Hum Reprod 1998; 13: 1014-19.

26- Jacobs M, Stolwijk AM, Wetzels AM. The effect of insemination/ injection time on the results of IVF and ICSI. Hum Reprod 2001; 16: 1708-13.

27- Nagy ZP, Liu J, Joris $H$, Devroey P, Van Steirteghem AC. Time-course of oocyte activation, pronucleus formation and cleavage in human oocytes fertilizaed by intracytoplasmic sperm

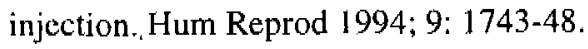

28- Chen C and Kattera S. Rescue ICSI of oocytes that failed to extrude the second polar body $6 \mathrm{~h}$ post-insemination in conventional IVF. Hum Reprod 2003; 18: $2118-21$.

29- Tesarik J. Associate Editor's comment on "Rescue ICSI of oocytes that failed to extrude the second polar body $6 \mathrm{~h}$ post-insemination in conventional IVF" by Chen and Kattera. Hum Reprod 2003; 18 : 2122-23. 